\title{
Research on the Impact Test and Control Countermeasures of Residential Elevator Operation Noise
}

\author{
Peng Hong ${ }^{1, *}$, Lv Zhong ${ }^{2}$, and Chen Mingfeng ${ }^{3}$ \\ ${ }^{1}$ Chongqing Jian Zhu College, Chongqing 400070, China; \\ ${ }^{2}$ Chongqing Construction Science Research Institute, Chongqing 400050, China; \\ ${ }^{3}$ Chongqing University, Chongqing 400030, China
}

\begin{abstract}
The influence of the residential elevator operating noise on the indoor personnel of the adjacent elevator room is becoming more and more prominent. Through on-site testing of a household in Chongqing affected by elevator noise, the noise distribution in key areas affected by elevator noise was experimentally studied, and compared with the current standard limits. Combined with the noise spectrum characteristics, the experimental analysis obtained the noise spectrum diagrams of different areas such as elevator machine rooms, corridors and bedrooms. It is believed that low-frequency noise has a greater impact on the residential acoustic environment. Combined with the noise propagation path of elevators, the technical measures and improvement schemes for noise reduction of elevator equipment operation noise are proposed, which can provide reference for the design and improvement of residential acoustic environment.
\end{abstract}

\section{Preface}

With the rise of high-rise buildings, the use of elevators has become increasingly widespread and has become one of the most frequent special equipment in various buildings. Because of its manned function, elevator operation has put forward stricter requirements on its reliability, vibration, noise and comfort. The "Elevator Technical Conditions" GB/T 10058-2009 clearly stipulates that for residential elevators with a rated speed less than or equal to $2.5 \mathrm{~m} / \mathrm{s}$, the maximum noise value in the elevator car during operation should not exceed $55 \mathrm{~dB}$; for residential elevators with speeds between 2.5 and $6 \mathrm{~m} / \mathrm{s}$, the maximum noise value in the elevator car during operation should not exceed $60 \mathrm{~dB}$; during the door opening and closing process of all residential elevators, the maximum noise value should not exceed $65 \mathrm{~dB}$. Due to the unreasonable design, construction or use of the elevator, the vibration and noise generated by the elevator during operation will diffuse to the surrounding rooms through the walls and floors of the building in a solidborne sound transmission mode. The resulting elevator noise will affect the indoor acoustic environment quality of the building and endanger people's physical and mental health ${ }^{[1-3]}$. In recent years, there have been more and more complaints about elevator noise in high-rise residential buildings. Therefore, the prevention and control of residential elevator noise has caused widespread concern in the society ${ }^{[4]}$. In this paper, combined with the actual measurement of specific engineering cases, the characteristics of elevator noise are analyzed, and its control methods and technical measures are discussed, so as to provide reference for the management of elevator noise in high-rise residential buildings.

\section{The source and characteristics of elevator noise}

\subsection{The source of elevator noise}

During the operation of the elevator, noise will be generated due to the rotation of the traction machine, the opening and closing of the door, the attraction and release of the relay and the contactor, the friction between the guide rail and the guide shoe, etc. This kind of noise is transmitted in the building through the components supporting the elevator and directly affects people's life and work. According to the different nature of the noise generated by elevator operation, it is mainly divided into three types: mechanical noise, aerodynamic noise and electromagnetic noise.

(1)Mechanical noise. There are three main sources of mechanical noise: First, friction and impact occur on the rotating part of traction motor in the machine room, centrifugal force caused by excessive dynamic balance deviation of brake, friction between meshing surfaces of worm gear of reducer and sliding friction between traction wheel and steel wire rope, all of which will produce mechanical noise. Second, mechanical noise is also caused by the following situations ${ }^{[5-6]}$ : high-speed rotation of the rope sheaves on the top of the elevator car in the hoistway, loose elevator car fasteners or unreasonable installation positions and quantities, vibration caused by the vibration frequency of the elevator car wall being close to the

\footnotetext{
${ }^{*}$ Corresponding author: penghong2952@163.com
} 
system vibration frequency, friction between the elevator car and the counterweight when running up and down along the guide rail, due to the problem of guide rail installation, the vibration of the elevator car and the counterweight during operation, the friction between the guide shoe and the guide rail, the insufficient preload of the guide shoe spring or the unreasonable intermittent between the guide shoe and the guide rail surface, etc. Third, when the elevator hall door and car door are opened and closed, the action sound of the interlocking mechanism and the collision sound of the safety contact plate will also produce mechanical noise.

(2) Aerodynamic noise. When the elevator car is running in the hoistway, as the speed of the box body increases, the gas is compressed sharply, and the hoistway air is disturbed up and down and airflow collision occurs. At this time, a lot of aerodynamic noise will be generated, and even pressure waves will be generated. At the same time, the flow velocity of the gas in the gap between the outer wall of the elevator car and the wall of the hoistway increases sharply due to the sudden reduction of the flow area, which will produce a lot of aerodynamic resistance, and even separation and vortexes may occur at the upstream and downstream of the box. This tunnel-like aerodynamic effect also has a certain impact on the aerodynamic noise $\mathrm{e}^{[7]}$.

(3) Electromagnetic noise. The electromagnetic force that changes in time and space between the various parts of the elevator motor, as well as the unbalanced unilateral magnetic pulling force generated by the eccentric effect of the motor stator and rotor, will cause electromagnetic noise. At the same time, the generation of electromagnetic noise is closely related to the slot fit, slot inclination and the stiffness of the motor structure ${ }^{[8]}$.

\subsection{The propagation characteristics and hazards of elevator noise}

Because the elevator unit is based on a steel frame, the vibration generated by the motor and brake cannot be sufficiently attenuated. The vibration is transmitted to the floor and wall through the pedestal, and then propagated through the rigid structure such as concrete floor, column and beam, and then radiates the noise when it reaches the residence. Among the sources of elevator noise, mechanical noise caused by vibration during elevator operation is the most obvious. Relevant studies have shown that this type of noise transmission is mainly in the form of low-medium frequency vibration, which is transmitted through solid media such as walls and floors ${ }^{[9]}$. Different from high-frequency noise, the low-frequency noise with frequency between $20-200 \mathrm{~Hz}$ attenuates slowly, and the sound wave is very long, which can easily pass through obstacles and enter the human ear. Because lowfrequency noise is mainly transmitted through the structure, it is easy to cause people's sensory resonance, so people's tolerance to low-frequency noise is relatively low.

Although the direct impact of low-frequency noise on human physiology is not as obvious as that of highfrequency noise, the impact on the overall physical and mental health of the human body is more significant. Due to the frequent operation of residential elevators, people will be exposed to the pollution of low-frequency elevator noise for a long time. Their auditory system, nervous system, cardiovascular system, and digestive system will be significantly damaged, mainly as tinnitus, earache, tympanic membrane damage, sympathetic nervousness, upset, grumpy, blurred vision, etc. At the same time, the low-frequency noise of elevators can cause disorders of internal secretion and decreased immune function, and increase the consumption of various vitamins, amino acids, glutamic acid, lysine and other nutrients in the human body. This has a great impact on the growth and development of the human body, especially children. Secondly, low-frequency noise is particularly harmful to pregnant women and fetuses, causing uterine contractions in pregnant women, affecting the blood supply of the fetus, and then affecting the development of the fetal nervous system. Generally speaking, pregnant women, children, the elderly, people with chronic diseases, and people with sensitive physique are more susceptible to low-frequency noise, and the damage is more serious ${ }^{[10-12]}$.

\section{Noise measurement of residential elevator}

\subsection{Case overview}

A resident on the 24th floor of a community in Chongqing reported that the elevators produced significant noise during the start, stop and operation, and they were more prominent at night, which seriously affected the daily life of the residents. This paper takes this residence as the test object, and conducts on-site measurement of residential elevator operation noise. The residence is a $2+1$ split-level on the top floor, and the unit type is shown in Figure 1. The black boxed area in the figure was originally an outdoor area, and the resident was transformed on the basis of the original construction drawings. The $1 \mathrm{~F}-3 \mathrm{~F}$ black-framed areas were transformed into balconies, kitchens, bedrooms and corridors, and the transformed areas were close to the elevator shaft and elevator machine room. According to residents' descriptions, this area is significantly affected by elevator noise, and the $2 \mathrm{~F}$ bedroom is the most affected.

The reasons for the excessive indoor noise of the residents were preliminarily judged on the spot. One is that the elevator machine room is separated from the bedroom by a wall, and the wall is made of blocks with poor sound insulation; the other is the rigid connection between the elevator load-bearing steel frame and the building structure. The solid noise generated by the elevator is transmitted to the lower floor through the main engine base, and then transmitted from the floor to the adjacent bedroom walls and rooms, causing solid and metal sound transmission, and finally causing the indoor noise of the residents to exceed the standard.

\subsection{Testing method}

According to the relevant standards, the testers 
respectively selected four locations as inspection points after site surveys, as shown in Figure 1 (b) and (c). The daytime when the elevator runs frequently is selected as the test time period, and the CESVA building sound insulation test system with a test range of $23 \mathrm{~dB}-140 \mathrm{~dB}$ and an accuracy of $\pm 0.7 \mathrm{~dB}$ is used as the test tool to continuously detect elevator noise. The test points layout description are shown in Table 1.
Table1. Description of test points layout

\begin{tabular}{cccc}
\hline test point 1 & test point 2 & test point 3 & test point 4 \\
\hline transformed & bedroom \\
living room & $\begin{array}{c}\text { renovated corridor } \\
\text { (abbreviated as " } \\
\text { "bedroom") }\end{array}$ & $\begin{array}{c}\text { elevator } \\
\text { machine room }\end{array}$ \\
& corridor ") & \\
\hline
\end{tabular}
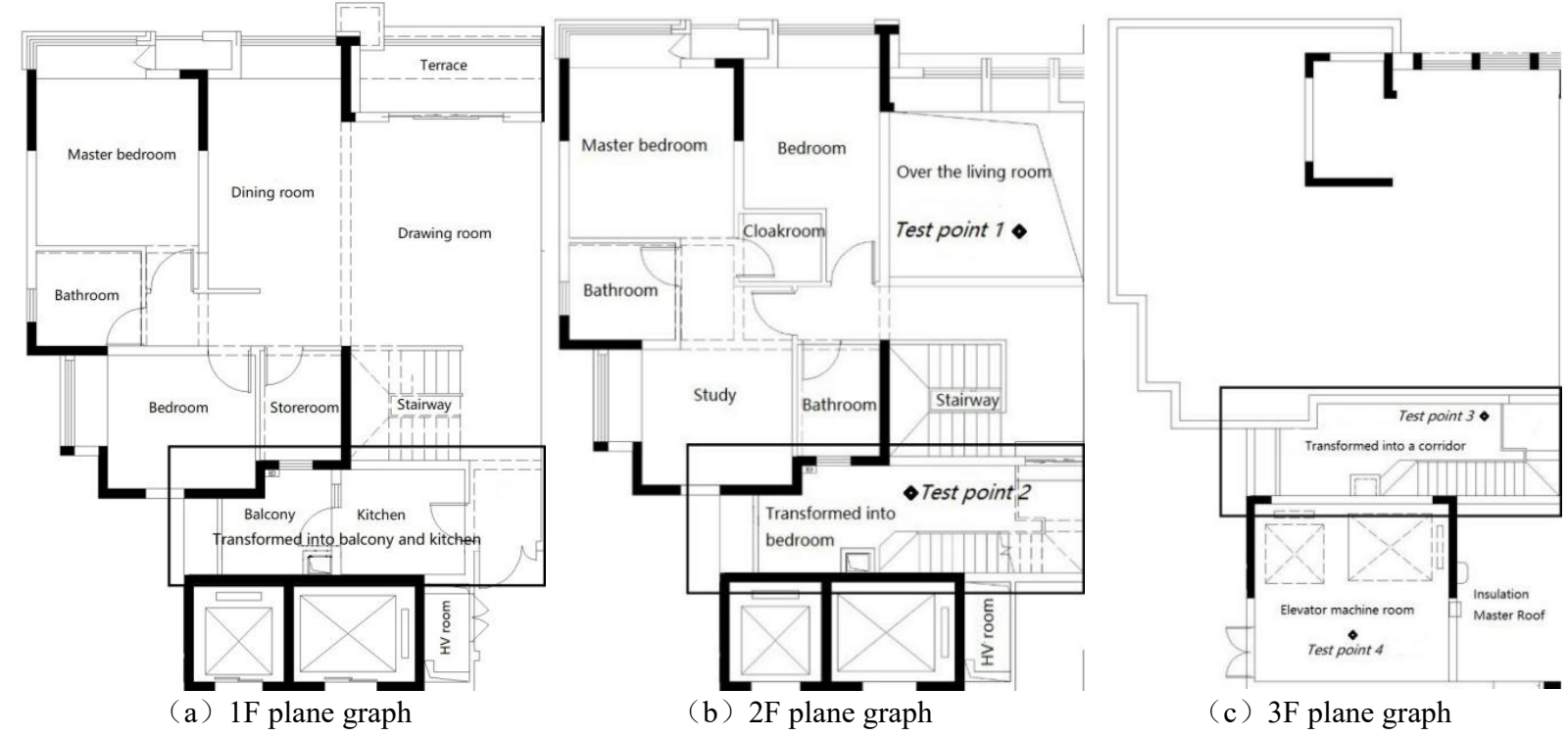

Fig1. The plane graph of the residential in the case

(c) 3F plane graph

\section{Results and analysis}

\subsection{Comparative analysis of test results and standard values}

It can be seen from Figure 1 that the test point in the living room area is closer to the outdoors. Normally, this area should be more affected by the outdoor acoustic environment. The test point in the corridor area is closer to the indoor (elevator shaft) and should be less affected by the outdoor acoustic environment. However, according to the test data in Table 2, the noise of the corridor test point is higher than that of the living room test point, so it can be determined that the noise mainly comes from the elevator machine room. The bedroom and the corridor are close to the elevator shaft and the elevator machine room respectively, and the test data shows that the noise value of the corridor is significantly higher than the noise value of the bedroom. This phenomenon shows that the mechanical noise generated by the vibration of the equipment in the elevator machine room has a significantly higher impact on the adjacent rooms than the aerodynamic noise in the elevator shaft.

At the same time, it can be seen from Table 2 that the noise value of the $250 \mathrm{~Hz}$ frequency band in the octave band sound pressure level in the daytime corridor area exceeds the standard. The specific performance is: the standard value is $44 \mathrm{~dB}$, and the test value is $48.3 \mathrm{~dB}$ (nonmandatory index), which exceeds the standard by $4.3 \mathrm{~dB}$. Although the night noise test has not been carried out, the noise standard value at night is lower than that during the day, so it can be determined that when the elevator is operating at night, there should be a number of low frequency noise values exceeding the standard. It can be seen from Table 2 that the influence of background noise is also significant, and the background noise values of the sound pressure level in octave bands are close to the noise test value. Among them, the background noise value of the corridor area in the $31.5 \mathrm{~Hz}$ frequency band is significantly higher than the noise test value. After residents fall asleep at night, the impact of background noise on human sleep will be more obvious.

Table2. Comparison table of field test data and standard value

\begin{tabular}{|c|c|c|c|c|c|c|c|c|c|}
\hline \multirow{2}{*}{\multicolumn{2}{|c|}{ test points }} & \multirow{3}{*}{$\begin{array}{c}\text { test } \\
\text { period }\end{array}$} & \multirow{2}{*}{$\begin{array}{l}\text { the type } \\
\text { of the } \\
\text { room }\end{array}$} & \multirow{2}{*}{ 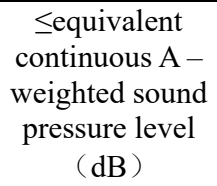 } & \multicolumn{4}{|c|}{$\leq$ sound pressure level in octave bands } & \multirow{2}{*}{$\begin{array}{l}(\mathrm{dB}) \\
500 \mathrm{~Hz}\end{array}$} \\
\hline & & & & & $31.5 \mathrm{~Hz}$ & $63 \mathrm{~Hz}$ & $125 \mathrm{~Hz}$ & $250 \mathrm{~Hz}$ & \\
\hline \multirow{2}{*}{\multicolumn{2}{|c|}{ standard value }} & & bedroom & 45.0 & 76.0 & 59.0 & 48.0 & 39.0 & 34.0 \\
\hline & & daytime & $\begin{array}{l}\text { living } \\
\text { room }\end{array}$ & 45.0 & 79.0 & 63.0 & 52.0 & 44.0 & 38.0 \\
\hline \multirow{2}{*}{$\begin{array}{l}\text { test point } 1- \\
\text { living room }\end{array}$} & test noise & \multirow{2}{*}{ daytime } & \multirow{2}{*}{$\begin{array}{l}\text { living } \\
\text { room }\end{array}$} & 31.0 & 42.8 & 41.7 & 43.0 & 38.5 & 27.0 \\
\hline & $\begin{array}{c}\text { values } \\
\text { background }\end{array}$ & & & 27.4 & 37.5 & 35.3 & 34.0 & 27.8 & 20.6 \\
\hline
\end{tabular}




\begin{tabular}{|c|c|c|c|c|c|c|c|c|c|}
\hline & $\begin{array}{l}\text { noise values } \\
\text { correction } \\
\text { values }\end{array}$ & & & 28.0 & 40.8 & 38.7 & 42.0 & 38.5 & 26.0 \\
\hline \multirow{4}{*}{$\begin{array}{l}\text { test point } 2- \\
\text { bedroom }\end{array}$} & $\begin{array}{l}\text { test noise } \\
\text { values }\end{array}$ & & & 31.0 & 42.8 & 41.7 & 43.0 & 38.5 & 27.0 \\
\hline & $\begin{array}{l}\text { background } \\
\text { noise values }\end{array}$ & daytime & bedroom & 27.4 & 37.5 & 35.3 & 34.0 & 27.8 & 20.6 \\
\hline & $\begin{array}{l}\text { correction } \\
\text { values }\end{array}$ & & & 28.0 & 40.8 & 38.7 & 42.0 & 38.5 & 26.0 \\
\hline & $\begin{array}{l}\text { test noise } \\
\text { values }\end{array}$ & & & 41.1 & 52.6 & 42.6 & 45.8 & 48.3 & 37.5 \\
\hline \multirow[t]{2}{*}{$\begin{array}{l}\text { test point } 3- \\
\text { corridor }\end{array}$} & $\begin{array}{l}\text { background } \\
\text { noise values }\end{array}$ & daytime & $\begin{array}{l}\text { living } \\
\text { room }\end{array}$ & 27.4 & 54.0 & 38.1 & 34.0 & 27.8 & 23.0 \\
\hline & $\begin{array}{l}\text { correction } \\
\text { values }\end{array}$ & & & 41.1 & 52.6 & 42.6 & 45.8 & 48.3 & 37.5 \\
\hline $\begin{array}{l}\text { test point } 4- \\
\text { elevator } \\
\text { machine room }\end{array}$ & $\begin{array}{l}\text { test noise } \\
\text { values }\end{array}$ & daytime & - & 70.8 & 48.0 & 57.2 & 65.2 & 73.8 & 71.4 \\
\hline
\end{tabular}

Note: The standard value comes from "Social Life Environment Noise Emission Standard" GB 22337-2008.

\subsection{Spectrum Energy Analysis of Elevator Operation Noise}

Only by deeply mastering the spectrum characteristics of noise sources can noise control be carried out more effectively. This paper analyzes the noise spectrum energy of the elevator machine room, corridor and bedroom area during the three periods of elevator start, operation, and stop. The results are shown in Figure 2-Figure 4.

From Figure 2-Figure 4, we can see that the noise spectrum energy intensity of the elevator machine room is mainly concentrated in the $200-500 \mathrm{~Hz}$ region, and the frequency is divided in the intermediate frequency range; the noise spectrum energy intensity of the corridor area is mainly concentrated in the $125-250 \mathrm{~Hz}$ region, and the frequency is divided in the low frequency range; the energy intensity of the noise spectrum in the bedroom area is mainly concentrated in the $60-150 \mathrm{~Hz}$ area, and the frequency is divided in the low frequency range. The noise spectrum energy intensity of the corridor and bedroom area is relatively low, and a small amount of frequency intensity below $60 \mathrm{~Hz}$ (ultra low frequency) appears. Therefore, from the perspective of spectral energy intensity, the residence is mainly affected by the low-medium frequency $(60-250 \mathrm{~Hz})$ noise generated during elevator operation. The resident is exposed to the low-medium frequency noise environment caused by elevator operation for a long time, and his physical and mental health will inevitably be greatly damaged.
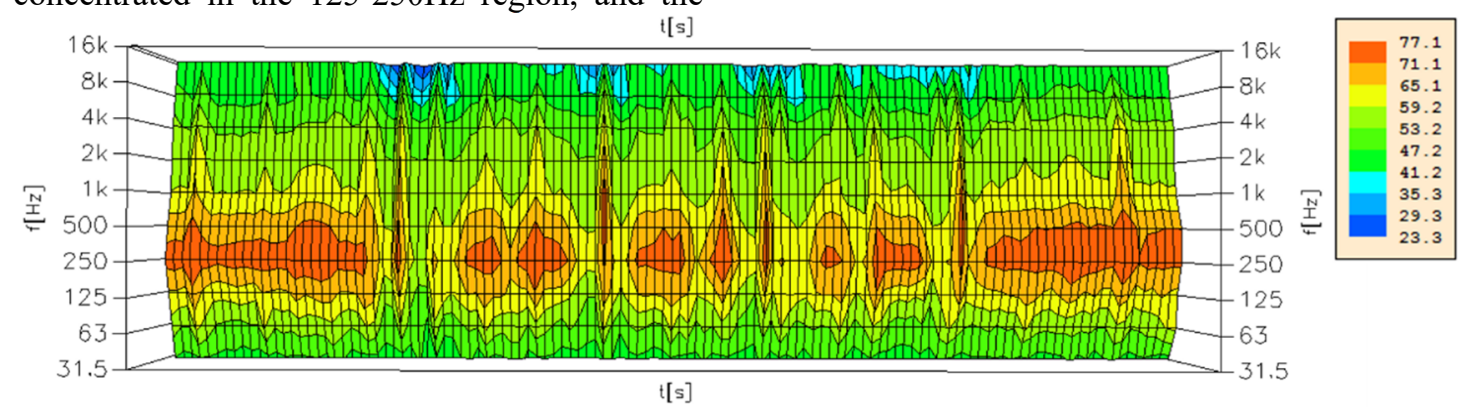

Fig2. Noise spectrum energy diagram of the elevator machine room

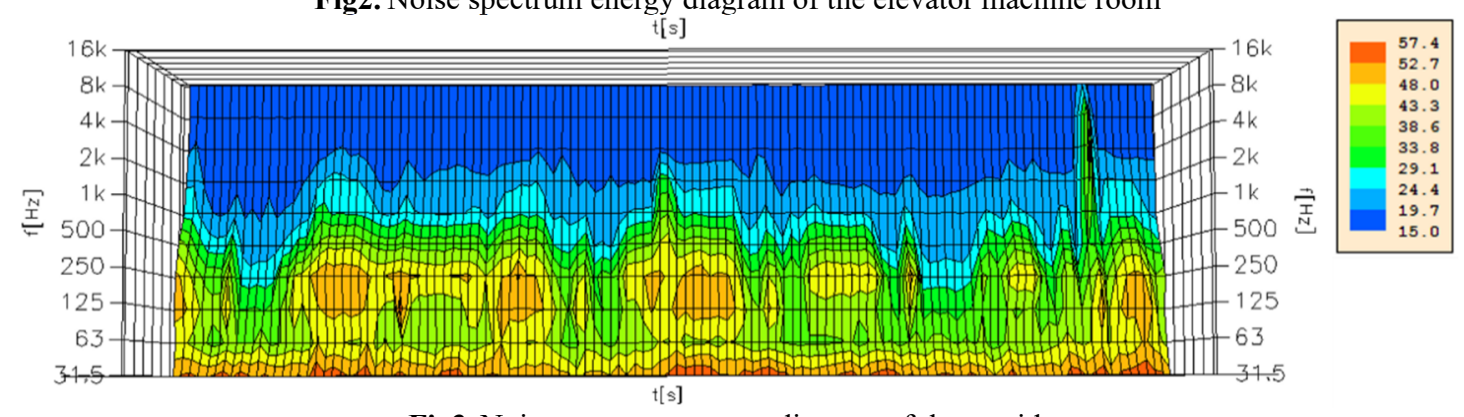

Fig3. Noise spectrum energy diagram of the corridor 


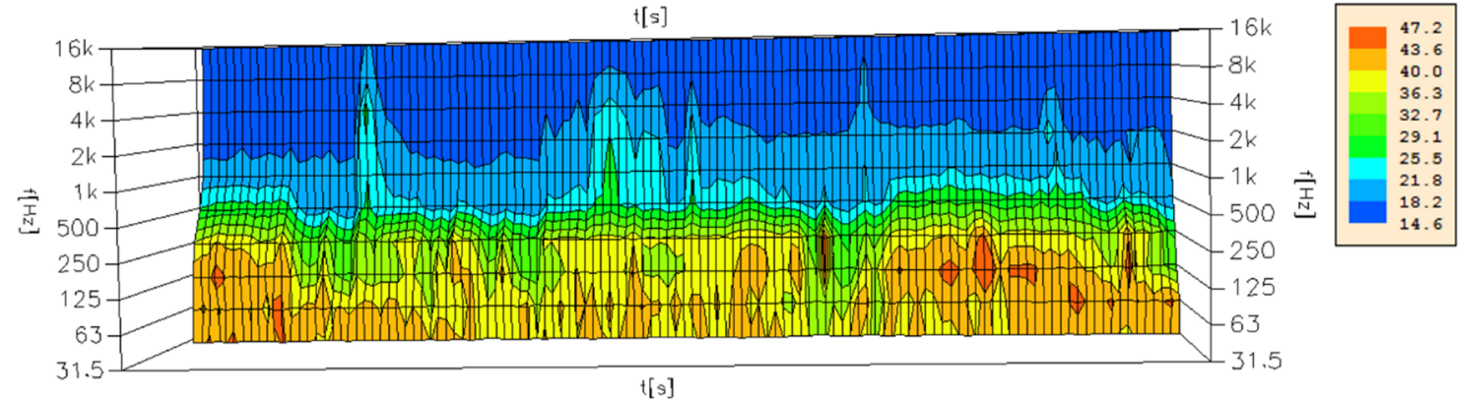

Fig4. Noise spectrum energy diagram of the bedroom

However, the current existing noise evaluation methods underestimate the actual impact of lowfrequency noise components on people. At present, our country's current acoustic environmental quality standard system mainly uses equivalent A sound level to test and evaluate environmental noise. The frequency characteristic of A-weighting is that the low frequency and high frequency of noise are greatly attenuated. Therefore, when the equivalent $\mathrm{A}$ sound level is used to test the elevator noise, the sound level of low-frequency noise has been greatly attenuated, resulting in the detection instrument not being able to accurately display the noise in this frequency band. As a result, when most of rooms which are adjacent to elevator machine rooms or elevator shaft use conventional instruments to test the indoor noise environment, the test results do meet the national standard values, but in fact, residents still suffer from elevator noise pollution for a long time. This is also true of the feedback from the households tested in this paper. For this reason, the authors believe that as people's requirements for the quality of the living environment increase, the equivalent A sound level evaluation method has certain limitations, and environmental testing standards and testing methods for low-frequency noise should be further formulated.

\section{Noise reduction measures}

The methods of controlling noise mainly include controlling the noise source, cutting off the transmission route and protecting the spreader. The medium and low frequency noise of the elevator is mainly generated by the vibration of the equipment, and then spreads through the building structure, especially through the walls and floors with poor sound insulation. In order to reduce the influence of elevator noise, the authors propose the following technical measures.

(1) The installation quality of the elevator traction machine will directly affect the noise decibel value of the elevator operation. The part where the traction machine base is connected to the I-beam load-bearing beam or concrete foundation should adopt spring shock absorbers, rubber shock absorbers or sound-absorbing sponges to achieve vibration reduction and adjustment. The load end of the traction sheave shaft is only allowed to have an upward inclination angle. At the same time, the channel formed by the traction rope through the floor will further enhance the noise propagation. Elevator noise is introduced into the elevator shaft through this channel, and then affects the rooms around the elevator shaft and the elevator car. It is recommended to install a muffler or sound isolator at a suitable position under the floor ${ }^{[14]}$.

(2) Improving the sound insulation performance of the wall in the elevator machine room is an effective way to reduce elevator noise. It can be achieved by using light steel keel to fix sound-absorbing cotton or spraying new sound-absorbing materials on the surrounding walls of the elevator machine room, and then installing soft and porous sound-absorbing panels or asbestos sound-proof panels on the outer layer. In particular, it is necessary to install a board with both sound insulation and sound absorption functions on the wall of the elevator machine room near the side of the residential room, and fill the wall with dense sound insulation material. This can not only increase the sound insulation effect of intermediate frequency noise, but also absorb the energy of low frequency noise and reduce intermediate frequency reverberation noise.

(3) The control of aerodynamic noise in the elevator shaft should not be underestimated. The distance between the elevator hoistway wall and the outer wall of the elevator car should be fully considered, and vents or ventilation equipment should be arranged reasonably. At the same time, sound absorption and sound insulation processing devices can be installed in the elevator hall and car to reduce the aerodynamic noise caused by wind pressure in the elevator shaft during operation.

\section{Conclusion}

Through the analysis of the characteristics of elevator noise and the actual measurement of elevator operating noise, the following conclusions are obtained from three aspects: building design, detection and control of lowfrequency noise generated by elevator vibration, and daily operation management.

(1) Elevator noise mainly comes from mechanical noise caused by equipment vibration during operation. This kind of noise is low-medium frequency noise, and the physical and mental health of people exposed to elevator noise for a long time will be seriously affected.

(2) Elevator noise has a particularly serious impact on the rooms which are adjacent elevator machine rooms or elevator shaft rooms. From the noise spectrum point of view, the noise frequencies affecting households are mainly concentrated in $60-250 \mathrm{~Hz}$. There are still shortcomings in noise detection and evaluation, and it is necessary to further develop acoustic environmental quality standards for low-frequency noise.

(3) Strengthening the maintenance of elevators is also conducive to controlling elevator noise. For example, add lubricating oil to all mechanical parts in time to reduce 
friction, and tighten loose parts and screws to reduce vibration. This can not only ensure the safe operation of the elevator, but also reduce the influence of elevator noise.

\section{Acknowledgments}

Chongqing Education Commission Science and Technology Research Project ( KJQN202004302 )

"Research on Water Resistance and Strength Performance of Large Volume Cement-Desulfurized Gypsum Composite Cement System"

\section{References}

1. Tianshui Guo. Vibration and noise analysis of highspeed traction elevator. Master's thesis, Guizhou University, China, 2019 (in Chinese).

2. Xiaowei Wang, Yanjie Yu, Ruijun Zhang, et al. Noise and Vibration Control, 2014, 34(3), 1(in Chinese).

3. Baek, Kwang-Hyun, Kim,et al. Transactions of the Korean Society for Noise and Vibration Engineering, 2011, 21(1).

4. Wenhuan Qiu. China Elevator, 2019, 30(12), 49(in Chinese).

5. Jicun Wang, Menghua Chen. Safety Technology of Special Equipment, 2020, (5), 41(in Chinese).

6. Zhi Xu. China Elevator, 2018, 29(18), 45(in Chinese).

7. Xiaolin Zhang, Jingwei Ji . China Elevator, 2018, 29(18), 43(in Chinese).

8. Yanbin Yin, Hongliang Zhang. Mechanical \& Electrical Engineering Technology, 2019, 48(11), 240(in Chinese).

9. Zhengxin Cai, Xuhong Guo. Value Engineering, 2019, (30), 161(in Chinese).

10. Xiaohua Teng, Yongxin Yao, Da Duan, et al. Military Medical Journal of South China, 2015, 29(9), 660(in Chinese).

11. Jie Yin. Environmental Study and Monitoring, 2017, 30(2), 56(in Chinese).

12. Bin Liu. Science and Technology \& Innovation, 2016, (21), 104(in Chinese).

13. Shiguang Cui. Environmental Protection and Circular Economy, 2019, (7), 61(in Chinese).

14. Yanqiu Chen, Lizhong Yang, Zhijian Fu, et al. Building Simulation, 2018, 11(4). 